\title{
Peer support versus interpersonal group therapy for prenatally depressed women
}

\begin{abstract}
In a previous comparison prenatally depressed women in a peer support group versus an interpersonal therapy group had similar decreases on depression and anxiety scale scores but lower cortisol levels by the end of the treatment period. For the current study, the videotapes that were made of the group sessions during the previous study were coded for affect, talking and discussion themes. Analyses of these data suggested that the peer support group had more frequent positive effect, talked more, especially at the same time (many voices), and talked about their expected infant more often. The therapy group members more often took turns talking and talked more frequently about work. These data support the earlier self-report and cortisol data suggesting that peer support group sessions may be a cost-effective form of intervention for prenatal depression.
\end{abstract}

Volume 9 Issue 4 - 2018

\author{
Field Tiffany $M$ \\ Department of Pediatrics, University of Massachusetts, USA
}

Correspondence: Field Tiffany M, Department of Pediatrics, University of Massachusetts, USA, Email tfield@med.miami.edu

Received: August 09, 2018 | Published: August 22, 2018

\section{Impact}

The novel finding of this study is that prenatally depressed women showed more positive effect, talked more and talked more about their expected infant in peer support groups versus interpersonal therapy groups. This suggests that peer support groups can be a cost effective form of intervention for prenatally depressed women.

\section{Peer support versus interpersonal group therapy for prenatally depressed women}

In a meta-analysis on perinatal depression treatments, interpersonal psychotherapy had greater effect sizes when compared to those of the control groups. ${ }^{1}$ At least two studies have documented the positive effects of interpersonal psychotherapy on prenatally depressed women., ${ }^{2,3}$ In a more recent study, comparisons were made between a peer support and an interpersonal psychotherapy group for prenatally depressed women. ${ }^{4}$ In this study, 44 prenatally depressed women were randomly assigned at 22 weeks gestation to one of these groups. The interpersonal psychotherapy group participated in one hour long sessions each week for three months while the peer support group met for only 20 minutes each week for three months. Self-report and cortisol measures were taken before and after the sessions. Although the peer support group had a lower socioeconomic status and met for shorter sessions, both groups had lower depression and anxiety scores by the end of the treatment period. Cortisol levels also decreased after the last session for both groups. However, the decrease was greater for the peer support group. These data suggested that peer support group sessions may be a cost-effective therapy for prenatally depressed women given their shorter sessions and the absence of a trained group psychotherapist. The purpose of the present study was to code and analyze the videotape data from the Field et al., ${ }^{4}$ study. Only selfreport and cortisol data were reported in that paper. In this study, positive effect, the type of talking (taking turns or talking at the same time) and discussion themes were coded to further assess the peer support group versus the interpersonal group therapy effects on the prenatally depressed women.

\section{Methods}

\section{Participants}

As already described in Field et al., ${ }^{4} 44$ depressed pregnant women were recruited from two prenatal ultrasound clinics (recruitment sample $=182)$ at a large university medical center at 20-24 weeks gestation ( $\mathrm{M}=22$ weeks) and randomly assigned to a peer support or an interpersonal psychotherapy group. The recruitment criteria were:

1. Prenatal depression as diagnosed on the Structured Clinical Interview for Depression

\section{Being primiparous}

3. Having an uncomplicated pregnancy

4. Being less than 40-years-old

5. No drug use (i.e., prescribed or illicit).

Previous or concurrent therapies were not exclusion criteria given that women previously recruited at these prenatal clinics had a very low incidence (3-5\%) of treatment for prenatal depression (i.e., psychotherapy or antidepressants). The women were primarily low income and Hispanic or African-American women with a high-school education and ranged in age from 18 to 40 years (M=25years) (See Table 1 for demographic data). The peer support group had a higher SES score, meaning they were lower income, and they had a higher baseline depression score, suggesting they were at greater risk than the interpersonal psychotherapy group.

Table I Means (and standard deviations in parentheses) on demographic variables for psychotherapy and peer support groups ${ }^{4}$

\begin{tabular}{|c|c|c|c|}
\hline \multirow{2}{*}{ Variable } & \multicolumn{2}{|l|}{ Group } & \multirow{2}{*}{$\mathbf{P}$} \\
\hline & Psychotherapy & Support & \\
\hline Age & $25.7(5.3)$ & $24.1(5.05)$ & NS \\
\hline Education & $4.3(1.2)$ & $4.0(3.8)$ & NS \\
\hline SES & $4.1(1.2)$ & $4.7 \quad(.6)$ & 0.03 \\
\hline Ethnicity (\%) & & & NS \\
\hline African American & 87 & 85 & \\
\hline Hispanic & 12 & 14 & \\
\hline Non-Hispanic White & I & I & \\
\hline Marital Status (\%) & & & NS \\
\hline Single & 40 & 36 & \\
\hline Boyfriend & 32 & 50 & \\
\hline Married & 28 & 14 & \\
\hline
\end{tabular}




\section{Procedures}

The women in the peer support group attended 20-minute sessions once per week for 3 months. The members of this group discussed many different topics. Although a staff member was present, she was not a trained therapist and she was asked to not direct the group or actively participate. The Interpersonal Psychotherapy Group sessions were longer (one hour) and were also held each week for 3 months. They were led by a therapist who focused on pregnancy experiences and relationship problems. The curriculum for the Interpersonal Psychotherapy group was derived from the Comprehensive Guide to Interpersonal Psychotherapy (Weissman et al. 2000). The therapist was directive and active, not passive as in the peer support group The specific techniques taken from the Weismann et al 2000 guide included encouragement of affect, clarification, communication analysis, exploratory and behavior change techniques. The therapist had been trained in these techniques and received ongoing supervision. Three consecutive peer support groups ( $\mathrm{N}=8$ in each group) and three consecutive interpersonal therapy groups ( $\mathrm{N}=8$ in each group) to total 24 in each type of group met over the 3 month period. Two women dropped out of each of the groups to total 44 women across the 2 types of groups (peer support and Interpersonal group psychotherapy). Participants in both groups were paid $\$ 30$ for each session to compensate for childcare and transportation costs. Assessments were conducted at the beginning of the first group session ( $\mathrm{M}=22$ weeks gestation), and at the last group session ( $\mathrm{M}=34$ weeks gestation).

\section{Measures}

The Structured Clinical Interview for Depression (SCID) was given before the first group session for the diagnosis of depression, Before the first and the last group sessions the assessments included the Center for Epidemiological Studies Depression Scale, ${ }^{5}$ the State Anxiety Inventory ${ }^{6}$ and the State Anger Inventory. ${ }^{7}$ Saliva samples were collected before and after the first and last group sessions and were assayed for cortisol levels. The data for those measures are reported in Field et al., ${ }^{4}$ and were summarized above.

\section{Videotaping and coding}

The sessions were videotaped and the DVDs were subsequently coded second by second for positive effect, talking (one or many voices at a time as separate codes), references to the unborn infant (fetus), references to significant other, references to work, references to pregnancy problems and references to pregnancy resources. These categories were derived from a scanning by two researchers for frequent behaviors/topics of the videotapes. The videotapes were then coded second by second and the percentage time that the behaviors/ topics occurred and were submitted to group comparison ANOVAs. Notes were also made second by second of the more specific descriptors of the topics referred to in each of the categories, although these were not submitted to data analyses as there were too many variables for the sample size.

\section{Results}

As can be seen in Table 2, the group comparison ANOVAs revealed the following group differences. The interpersonal psychotherapy group as compared to the peer support group:

a. Engaged in more turn-taking (one voice talking at a time)

b. Talked more about work as a topic

The peer support group, in contrast to the interpersonal psychotherapy group:

i. Showed positive affect a greater percent of the time

ii. Talked over each other (multiple members talking at the same time)

iii. Talked about their unborn infant (fetus) a greater percentage of time

Table 2 Mean percentage time that behaviors/topics occurred across the group sessions (and standard deviations in parentheses).

\begin{tabular}{lllll}
\hline Variable & Psychotherapy & Peer support & F & P \\
\hline Positive affect & $24.85(I 7.1 \mathrm{I})$ & $59.30(2 \mathrm{I} .99)$ & 14.34 & 0 \\
One voice (taking turns) & $30.92(13.33)$ & $19.05(10.87)$ & 7.98 & $0.00 \mathrm{I}$ \\
Many voices at same time & $30.00(19.37)$ & $66.35(12.35)$ & 31.17 & 0 \\
Refer to unborn infant & $3.75(3.86)$ & $12.35(5.82)$ & 18.65 & 0 \\
Refer to significant other & $7.08(2.94)$ & $6.25(4.05)$ & N.S. & \\
Refer to work & $12.92(9.32)$ & $4.10(3.55)$ & 5.88 & 0.006 \\
Refer to pregnancy problems & $4.08(3.45)$ & $3.65(3.01)$ & N.S. & N.S. \\
Refer to pregnancy resources & $3.75(2.90)$ & $1.00(.97)$ & & \\
\hline
\end{tabular}

The references to the unborn infant in decreasing order of frequency were sex of the baby, ultrasound exam, preterm baby, fetal activity, baby shower, spoiling the baby, breast-feeding and baby names. The references to the significant other were support and relationship issues. The references to work were current work, loss of work and looking for work. The references to pregnancy symptoms in decreasing order of frequency were pain, weight gain, sleep, nausea, health risks/complications and food cravings. The decreasing order of frequency for pregnancy resources was type of delivery, father support, family support, social support and government assistance.

\section{Discussion}

The more positive affect and the greater talking at the same time that was coded from the videotapes for the peer support group suggest that these sessions without a trained therapist were more animated and may have been less stressful. This suggestion is supported by a decrease in cortisol being greater for the peer support group in the same sample as previously reported. ${ }^{4}$ This finding is consistent with other comparisons of clinician led versus peer support groups. For example, in one study, members of the clinician led group reported 
more hesitation about talking in the group and uncertainty about what to talk about. ${ }^{8}$ And, in another study, the participants described feeling less restricted and more comfortable in a peer led group and identified authority as a barrier to their interactions in the clinician led group. ${ }^{9}$ They viewed the peer led group as an opportunity to establish real connections with other people that were nonjudgmental. They expressed that empathy for each other was an important factor in the peer led group. They spoke to the researchers about opportunities to help and support each other in diverse ways and they enjoyed hearing the stories of their peers. The leaders of the peer group were seen as less skilled but were also found to be less threatening.

On the other hand, the members taking turns and not talking at the same time may have reflected more self-control related to the greater structure provided by the therapist. In the same study that reported more comfort speaking in peer led groups, professional leaders were perceived by the group members to be more knowledgeable and also able to keep the group on track. ${ }^{9}$

Although the groups' time spent on different discussion topics was very similar for the references to significant others and to pregnancy symptoms and pregnancy resources, the peer support group talked more frequently about their unborn infant and the interpersonal group psychotherapy members talked more about work. This may have related to the peer support group members all experiencing concerns about the unborn child, while the professional leader may have focused more on the conflicts members were experiencing about taking time off work during their pregnancy.

Surprisingly similar but small amounts of time were spent talking about the significant other, pregnancy symptoms and pregnancy resources relative to the focus on work by the therapist led groups and on the unborn infant in the peer support groups. It may be that these somatic symptoms and outside support issues were so common to all the members that they were taken for granted and not discussed. Alternatively, they may have been difficult to discuss and still remain feeling positive. Relatively speaking, positive affect (smiling and laughing) was notably frequent in both groups, although twice as frequent in the peer support group.

The clinical implications of these data are that peer support groups can be effective at keeping their members talking animatedly and experiencing positive feelings. This appears to be a form of costeffective therapy for prenatally depressed women as suggested by the study on self-report and cortisol data $^{4}$ and the current analyses of the videotaped discussions. Group members of other studies have discussed how the group provided a less expensive and more consistently available method for gaining help when their doctor or therapist was not available to them. ${ }^{8}$ And, peer support is shown to decrease patient psychiatric admissions, improve overall functioning and also reduce mental health treatment costs by providing less expensive than constant mental health treatment by therapists or psychiatrists. ${ }^{10}$ But, as some have suggested, peer support may be beneficial to certain individuals at certain times and may reduce certain symptoms, but traditional mental health treatment may also be necessary. ${ }^{11}$

\section{Acknowledgements}

None.

\section{Conflict of interest}

The author declares that there is no conflict of interest.

\section{References}

1. Sockol LE, Epperson CN, Barber JP. A meta-analysis of treatments for perinatal depression. Clin Psychol Rev. 2011; 31(5):839-849.

2. Field T, Deeds O, Diego M, et al. Benefits of combining massage therapy with group interpersonal psychotherapy in prenatally depressed women. Journal of Bodywork and Movement Therapies. 2009;13(4):297-303.

3. Spinelli MG, Endicott J. Controlled clinical trial of interpersonal psychotherapy versus parenting education program for depressed pregnant women. Am J Psychiatry. 2003;160(3):555-562.

4. Field T, Diego M, Delgado J, et al. Peer support and interpersonal psychotherapy groups experienced decreased prenatal depression, anxiety and cortisol. Early Hum Dev. 2013;89(9):621-624.

5. Radloff L. The CES-D scale: A self-report depression scale for research in the general population. Applied Psychological Methods. 1977;1:385-401.

6. Spielberger CD, Gorusch TC, Lushene RE. The State Trait Anxiety Inventory (STAXI). Consulting Psychologists Press. Palo Alto, CA. 1970.

7. Spielberger CD, Ritterband LM, Sydeman SJ. Assessment of emotional states and personality trait: measuring psychological vital signs. In: Clinical Personality Assessment: Practical Approaches. New York: Oxford University Press; 1995:38-52.

8. Behler J, Daniels A, Scott J, et al. Depression/bipolar peer support groups: Perceptions of group members about effectiveness and differences from other mental health services. The Qualitative Report. 2017;22(1):213-236.

9. Pallaveshi L, Balachandra K, Subramanian P, et al. Peer-led and professional-led group interventions for people with cooccurring disorders: A qualitative study. Community Ment Health J. 2014;50(4):388-394.

10. Cook JA, Copeland ME, Jonikas JA, et al. Results of a randomized controlled trial of mental illness self-management using wellness recovery action planning. Schizophr Bull. 2012;38(4):881-891.

11. Eisen SV, Schultz MR, Mueller LN, et al. Outcome of a randomized study of a mental health peer education and support group in the VA. Psychiatric Services. 2012;63(12):1243-1246. 